\title{
Le français langue diplomatique de la Sublime Porte : le cas de la légation ottomane de La Haye
}

\author{
Arzu Etensel Ildem
}

\section{(2) OpenEdition}

\section{Journals}

Édition électronique

URL : https://journals.openedition.org/dhfles/328

DOI : $10.4000 /$ dhfles.328

ISSN : 2221-4038

Éditeur

Société Internationale pour l'Histoire du Français Langue Étrangère ou Seconde

Édition imprimée

Date de publication : 1 janvier 2007

Pagination : 215-239

ISSN : 0992-7654

Référence électronique

Arzu Etensel Ildem, « Le français langue diplomatique de la Sublime Porte : le cas de la légation ottomane de La Haye », Documents pour l'histoire du français langue étrangère ou seconde [En ligne], 38/39 | 2007, mis en ligne le 14 décembre 2010, consulté le 27 mai 2021. URL : http:// journals.openedition.org/dhfles/328; DOI : https://doi.org/10.4000/dhfles.328

Ce document a été généré automatiquement le 27 mai 2021.

(c) SIHFLES 


\title{
Le français langue diplomatique de la Sublime Porte : le cas de la légation ottomane de La Haye
}

\author{
Arzu Etensel Ildem
}

1 La tradition de la diplomatie chez les Turcs remonte au Moyen Âge. Bien avant l'Empire ottoman, les Etats turcs qui l'ont précédé ont correspondu avec les pays alliés ou adversaires de leur région (Asie et Anatolie). Avant la conquête de Constantinople, la correspondance avec Byzance se faisait en grec, et après la conquête, le Sultan Mehmet écrivait au pape Pie II en latin (Meydan 2006: 250). Quand l'Empire ottoman s'est imposé comme une force turque et musulmane à l'ouest de l'Europe, en Anatolie, au Proche-Orient et sur la rive sud de la Méditerranée, la langue ottomane a dominé l'administration et la littérature et elle a fortement subi l'influence de l'arabe et du persan.

$2 \mathrm{Au} \mathrm{XVIII}{ }^{\mathrm{e}}$ siècle, l'Empire ottoman commence à sentir le besoin de s'ouvrir vers l'Occident. Il a besoin d'apprendre les nouvelles techniques militaires pour recouvrer sa suprématie dans la région. Les premières écoles d'ingénieurs (dans le cadre des études militaires) sont fondées dans la deuxième moitié du XVIII ${ }^{e}$ siècle. Le comte de Bonneval, officier militaire français, et le baron de Tott, diplomate et général français d'origine hongroise, entrent au service du Sultan. « Dans [les écoles d'ingénieurs] l'enseignement se faisait grâce à des livres scientifiques traduits en grande partie du français.» (Etensel Ildem 1997 : 59). Au début du XIXe siècle, le Sultan Mahmut II crée « le Bureau de traduction de la Sublime Porte ", et on envoie les jeunes étudiants de ce bureau en Europe et surtout en France. Vers le milieu de ce siècle, nous pouvons dire que le français s'est solidement établi comme langue étrangère, voire seconde chez les élites ottomanes.

3 Le présent travail a pour but de présenter quelques exemples de la correspondance diplomatique de la légation ottomane de La Haye, et de voir quel rôle y a joué le français. Simple étude de cas, il ne prétend pas arriver à une conclusion générale sur l'ensemble de la correspondance diplomatique ottomane. 
Les relations diplomatiques entre les Pays-Bas et l'Empire ottoman remontent au début du XVII ${ }^{e}$ siècle ${ }^{1}$. Alors que les Pays-Bas ont établi une ambassade permanente à Istanbul en 1612, le gouvernement ottoman attend jusqu'au milieu du XIX ${ }^{\mathrm{e}}$ siècle pour fonder une légation permanente à La Haye. Le premier envoyé, chef de légation auprès du Royaume des Pays-Bas, est Kostaki Karaca Bey qui a présenté ses lettres de créances à Guillaume III en janvier 1855. Entre 1855 et 1923, année de la fondation de la République de Turquie, neuf chefs de légation se succèdent à La Haye. Pendant une période qui va probablement jusqu'à la fin du XIXe siècle, le légat ottoman de La Haye est aussi accrédité à Stockholm. Les consulats ottomans d'Amsterdam et de Rotterdam encadrent la légation de La Haye et, vers 1880, vient s'y ajouter le consulat de Batavia, l'actuelle Djakarta, capitale des Indes néerlandaises.

Les documents que nous allons examiner se trouvent dans les archives de l'Ambassade de Turquie à La Haye. Les plus anciens datent de la fin des années 1870. La plupart des dossiers qui se trouvent dans ces archives portent des titres en français et cela jusqu'à l'époque républicaine. Quand les titres des dossiers ne sont pas en français, ils sont en turc-ottoman. Précisons que nous appelons turc-ottoman le turc écrit en caractères arabes. Ces documents peuvent être classés en quatre catégories :

1. La correspondance intérieure, c'est-à-dire la correspondance entre la Sublime Porte et la légation de La Haye et les consulats.

2. La correspondance extérieure, c'est-à-dire la correspondance entre la légation ottomane et les autorités néerlandaises.

3. La correspondance avec les tiers, c'est-à-dire les diverses communications de la légation ottomane des Pays-Bas.

4. Divers documents qui ne sont classables dans aucune des trois catégories précédentes.

\section{Correspondance intérieure}

6 La correspondance entre la légation et le ministère des Affaires étrangères ottoman se faisait en français. Dans les dossiers datant des années 1870 et 1880, nous avons trouvé un grand nombre de lettres rédigées en français. Notre premier exemple (texte 1) est une lettre envoyée par la Sublime Porte à Karadja Pacha qui se trouve à l'époque à Stockholm.

7 Soulignons quelques détails intéressants :

1. L'en-tête du papier se présente en français: Sublime Porte, Ministère des Affaires Etrangères.

2. Pour dater la lettre, on a utilisé le calendrier grégorien, le système de datation occidental. Dans l'Empire ottoman la datation était différente et se faisait selon l'Hégire.

3. Les numéros officiels de la lettre sont des chiffres occidentaux (en fait des chiffres arabes). Le système numéral était différent chez les Ottomans.

4. Le nom de Caradja Pacha est translittéré en français de façon à ce que la prononciation turque change le moins possible. Le procédé est le même avec la signature du ministre des Affaires étrangères. Cette façon de translittérer la langue turque en caractères latins a été utilisée dès la fin du XVIII ${ }^{e}$ siècle, notamment entre Hatice Sultane, la sœur de Selim III, et le peintre architecte français Melling.

5. Cette lettre est écrite à la main. A partir du début $d u X X^{e}$ siècle apparaissent les lettres dactylographiées aussi bien en français qu'en turc-ottoman. 

français des informations à ses consulats et les consulats répondaient dans la même langue. L'en-tête de la légation comme celui des consulats se présentait en français: « légation Impériale de Turquie », «Consulat Général de Turquie ». Notons que le terme «Turquie », qu'on utilise en français, n'était pour ainsi dire pas utilisé en turc, car pour les Turcs, le pays s'appelait l'Empire ottoman. Il arrivait que les consuls soient des Néerlandais, mais même quand le consul était un citoyen ottoman, la correspondance se faisait en français.

(texte 2) envoyée par le ministre des Affaires étrangères Abédine Bey à Mourad Effendi, l'envoyé à La Haye, montre que la correspondance privée se faisait aussi en français. Notons que le ministre Abédine ainsi que l'envoyé Mourad Effendi sont tous deux des Ottomans turcs et musulmans et qu'ils communiquent pourtant en français.

10 Le français et le turc-ottoman étaient parfois utilisés dans la même lettre, ce qui indique que les deux langues étaient des outils de communication à part égale.

11 Les articles parus dans la presse néerlandaise pendant l'occupation de l'Anatolie par les forces grecques, à propos des atrocités commises sur la population civile, ont été traduits en français et transmis à la capitale ottomane en cette langue. La documentation de la légation était composée de textes en français et en turc-ottoman.

Une lettre dactylographiée envoyée par la légation de La Haye à la Sublime Porte datée de 1921 montre que jusqu'à cette date une partie de la correspondance se faisait en français.

Les télégrammes étaient rédigés en français. Ceci est compréhensible étant donné que le système ne devait pas être adapté aux caractères arabes. Les télégrammes étaient envoyés de «Constantinople» et portaient le nom du destinataire: "ministère des Affaires étrangères ». Le nom de l'expéditeur était «le Légat ottoman ». Le français était la langue internationale du système télégraphique puisque les noms et les titres du destinataire et de l'expéditeur exprimés en français ne changeaient pas, quel que soit le pays (Allemagne ou Pays-Bas) de destination.

14 Les nouvelles les plus importantes pouvaient être transmises dans ces télégrammes: l'annonce de l'ouverture à Istanbul du parlement ottoman de 1908 et par conséquent le début de la seconde monarchie constitutionnelle; ou bien la déchéance du Sultan Abdülhamit (texte 3) et son remplacement par Mohammed V. Remarquons que les Turcs ne disent pas Mohammed mais Mehmet. Il semble qu'on ait favorisé la version européenne des noms turcs quand on écrivait en français.

Tout comme la correspondance entre le ministère et les légations, les communications entre légations se faisaient également en français, ainsi qu'en témoigne une lettre à entête de l'«Ambassade Impériale Ottomane » de Rome, datant de 1921, envoyée au ministre plénipotentiaire de La Haye (texte 4). L'annexe de cette lettre fait référence à une autre lettre écrite en français par un sujet ottoman résidant à Athènes. Ce citoyen ottoman se plaint du fait que le gouvernement hellénique enrôle de force dans l'armée hellénique les sujets ottomans d'origine grecque et il demande à l'ambassade ottomane d'agir auprès du gouvernement hollandais, car c'est la Hollande qui protège les intérêts de l'Empire ottoman en Grèce.

16 La section consulaire du ministère des Affaires étrangères ottoman possède un en-tête spécial: "Sublime Porte, Ministère des Affaires Etrangères, Direction Générale des 
Consulats, des Affaires Commerciales et de Contentieux", ce qui montre que la correspondance consulaire se fait aussi en français.

Le Consulat ottoman de Batavia est très actif et montre que les Ottomans suivent de près les agissements des Néerlandais en Indonésie. En 1910, le gouvernement néerlandais décide de restreindre le séjour et les déplacements des étrangers d'origine asiatique dans les Indes néerlandaises. Le gouvernement ottoman réagit énergiquement à cette mesure car elle concerne une partie de ses sujets. Toute une correspondance en français témoigne de cette affaire. Aristarchi Bey, légat ottoman à La Haye et Erched Bey, consul général ottoman à Batavia, échangent un grand nombre de lettres à ce sujet (texte 5). La Sublime Porte demande incessamment à Aristarchi Bey de faire des démarches en vue d'une amélioration de l'état des sujets ottomans dans les Indes néerlandaises. Erched Bey met Aristarchi Bey au courant de la situation à Batavia et lui envoie la traduction en français du texte des ordonnances qui forme tout un livret.

18 Aristarchi Bey envoie à son ministère une longue lettre en français où il relate l'entretien mi-officiel mi-privé, qu'il a eu à ce sujet avec le ministre néerlandais des Affaires étrangères.

\section{Dossier spécial : condoléances} ottoman, probablement pour demander une faveur. Voilà qui est en contradiction avec l'image de la femme turque véhiculée par Aziyadé, le célèbre roman de Pierre Loti écrit à la même époque, dans lequel l'héroïne éponyme ne sait ni lire ni écrire. Assim Bey, le ministre ottoman des Affaires étrangères, affirme dans une lettre (texte 6) envoyée au chargé d'affaires que le placet de Madame Mourad Effendi sera placé sous les yeux de sa Majesté Impériale le Sultan par le «Grand Maréchal du Palais », en occidentalisant le titre ottoman de "baş mabeynci » qui était le dignitaire responsable des affaires privés du Sultan.

Les deuxième et sixième envoyés ottomans à La Haye, Mourad Effendi et Aristarchi Bey sont décédés en Hollande. Le premier en 1881 et le deuxième en 1914. L'un et l'autre ont été enterrés à La Haye. Sublime Porte qui est un accusé de réception, le ministre ottoman des Affaires étrangères exprime au chargé d'affaires Loghadès Effendi sa satisfaction à propos des honneurs rendus par le gouvernement néerlandais à l'occasion de la mort et des obsèques de Mourad Effendi.

Après la mort de son mari, Madame Mourad Effendi, a envoyé un placet au Sultan Une lettre rédigée par Talaat Bey, chargé d'affaires, annonce au consul général de Turquie à Batavia, la mort d'Aristarchi Bey en 1914. Aristarchi Bey était à La Haye depuis 1909. C'était un éminent diplomate ottoman d'origine grecque qui avait servi dans plusieurs légations ottomanes en Europe et aussi à Washington. C'est le même Grégoire Aristarchi qui a publié une série de cinq livres intitulés Législation ottomane, qui est le recueil des lois, règlements, ordonnances, traités, capitulations et autres documents de l'Empire ottoman promulgués entre 1839 et 1873 et traduits en français. Nous avons pu y vérifier que l'article 9 du règlement de l'École civile, qui est l'ancêtre 
de la faculté des Sciences politiques et qui à l'époque formait la presque totalité des fonctionnaires ottomans, dit que l'enseignement de la langue française est obligatoire. Il est toutefois stipulé que «L'enseignement de l'histoire universelle, de la géographie générale, du droit international, des mathématiques, de l'économie politique, de l'archéologie, de la physique et des autres leçons sera fait en langue turque.» (Aristarchi Bey $1874: 240$ )

Dans une longue lettre très émouvante, Talaat Bey, chargé d'affaires, relate à Said Halim Pacha, ministre des Affaires étrangères, les pompes de la cérémonie funéraire d'Aristarchi Bey (texte 7).

\section{Correspondance extérieure}

La lingua franca de la diplomatie européenne était le français, par conséquent les communications entre la Sublime Porte et les pays et ambassades étrangers se faisaient en français. Les notes verbales destinées à la légation ottomane étaient rédigées en français (texte 8).

La Maison royale des Pays-Bas avait toujours recours au français pour ses communications diplomatiques. De nos jours cette tradition n'a pas changé. Le ministère néerlandais des Affaires étrangères invitait la délégation ottomane à l'ouverture des États Généraux en français, et la partie ottomane répondait dans la même langue. De nos jours la Maison royale néerlandaise fait cette invitation au corps diplomatique se trouvant à La Haye en anglais et en français. La légation ottomane, puis à partir de la période républicaine, l'Ambassade de Turquie, a utilisé le français dans sa correspondance extérieure jusqu'aux années 1960, et puis, graduellement, l'anglais a remplacé le français dans la correspondance avec les pays et les ambassades étrangers.

Lors du contentieux à propos du séjour des sujets ottomans d'origine asiatique dans les Indes néerlandaises, un échange épistolaire a lieu entre le ministre néerlandais des Affaires étrangères et Aristarchi Bey, l'envoyé ottoman. Cet échange se fait en français. Nous voyons qu'il existe une relation très amicale entre les deux hommes (texte 9).

\section{Correspondance avec les tiers}

27 La légation ottomane avait recours au français dans plusieurs domaines concernant l'administration de la légation et la vie pratique. Les contrats de location entre le personnel de la légation et les propriétaires néerlandais se faisaient en français (texte 10). Les contrats passés entre les secrétaires recrutés localement et la légation ou le consulat (ottomans) étaient rédigés en français. Les affaires juridiques étaient également réglées en français. En 1918, un sujet ottoman, Joseph Chamay, décède à Scheveningen. La correspondance à propos de la succession Chamay se fait en français entre la légation et le bureau d'avocat des frères Brauw.

Certaines institutions internationales utilisent le français comme langue de communication. Dans une lettre datée de 1918, le Bureau de correspondance juif informe la légation ottomane des activités de la communauté juive de Palestine. La correspondance entre la légation et la Cour de justice internationale de La Haye se fait également en français. 
30 Le contentieux à propos du séjour des sujets ottomans d'origine asiatique a apparemment eu des répercussions dans l'Empire ottoman. Le directeur du journal Jeune Turc d'Istanbul, Djelal Noury Bey (translittération selon la prononciation française), s'inquiète de la situation des étrangers dans les Indes néerlandaises et demande des renseignements à Aristarchi Bey pour son ouvrage intitulé « le droit des gens à mon point de vue ». Cette lettre mise à la poste à Istanbul a été rédigée en français.

31 Le français se présente également comme un outil de communication entre la légation et les sujets ottomans qui se trouvent en Hollande et en Europe. Un avis aux sujets turcs est publié en français dans un journal néerlandais. Les ressortissants turcs conscrits des années 1316 (1900 selon de calendrier grégorien), 1317 (1901) et 1318 (1902) doivent se présenter à leur bureau de recrutement respectif en vue d'accomplir leur service militaire (texte 11). Une autre annonce en français parue dans un journal de Rotterdam, le Nieuwe Rotterdamsche Courant, le 15 avril 1920, annonce en français aux sujets ottomans l'amnistie accordée aux sous-officiers, caporaux et soldats qui ont déserté l'armée pendant la guerre. Le Nieuwe Rotterdamsche Courant du 8 février 1924 annonce que la Grande assemblée nationale de Turquie accorde une amnistie aux déserteurs des classes 1297-1315 (1881-1899) qui seront exemptés de service militaire ainsi qu'aux jeunes gens de la classe 1316-1317 (1900-1901) qui devront rentrer en Turquie pour accomplir leur service militaire. Cette fois il s'agit du Consulat général de la République de Turquie et non pas ottoman, car cela se passe après la fondation de la République (texte 12).

\section{Documents divers}

Les archives de La Haye contiennent également des documents rédigés en français ou en partie en français et qui ne font pas vraiment partie de la correspondance diplomatique.

Le règlement envoyé par la Sublime Porte en 1884, qui régit les passeports des ressortissants ottomans, est bilingue. Le livret se présente d'un côté en turc-ottoman et de l'autre en français. La partie en turc-ottoman n'a pas été entamée (les pages n'ont pas été coupées) tandis la partie en français porte des signes visibles d'usage répété (texte 13).

34 Les certificats de nationalité qui datent de 1928 sont également bilingues. Ce sont probablement les derniers documents officiels en turc-ottoman puisque la réforme de l'alphabet s'est faite en 1927 et qu'à partir de cette date la langue turque s'est écrite avec les lettres latines (texte 14).

35 La première liste diplomatique publiée en 1930 par le département du protocole du ministère des Affaires étrangères de la République de Turquie a été rédigée en français. Notons qu'Ankara s'appelle encore Angora et que les noms turcs sont translittérés selon la tradition dont nous avons déjà parlé.

36 A partir de la période républicaine les candidats à la diplomatie devaient passer un concours dont le règlement a été publié par le ministère des Affaires étrangères. Selon l'article 8 de ce règlement, les candidats étaient obligés de réussir un examen écrit et oral en français afin d'être acceptés au ministère. 


\section{Conclusion}

37 D'après les documents qui se trouvent dans les archives de l'ambassade de Turquie à La Haye, il semble bien que, pendant toute la période impériale, le français a été, à côté du turc-ottoman, la langue de communication dans l'ensemble de la correspondance diplomatique. La langue française était parfaitement maitrisée et utilisée comme une langue maternelle par les diplomates ottomans. Cette sophistication dans l'usage du français est remarquable quand on pense que l'Empire ottoman ne s'est ouvert à la culture occidentale qu'au début du XIX ${ }^{e}$ siècle.

Texte 1 : La Sublime Porte à Karadja Pacha

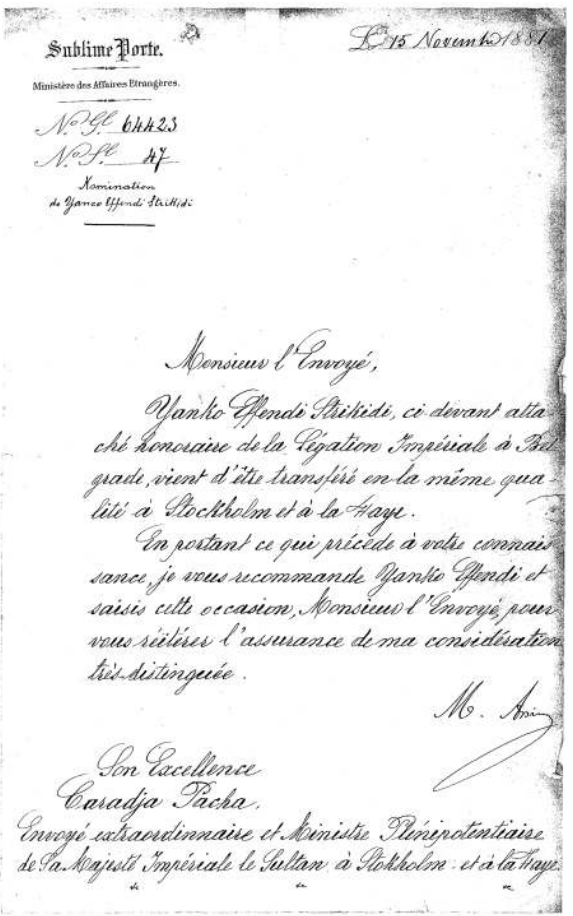

(1) 
Texte 2 : Le ministre des Affaires étrangères Abédine Bey à Mourad Effendi

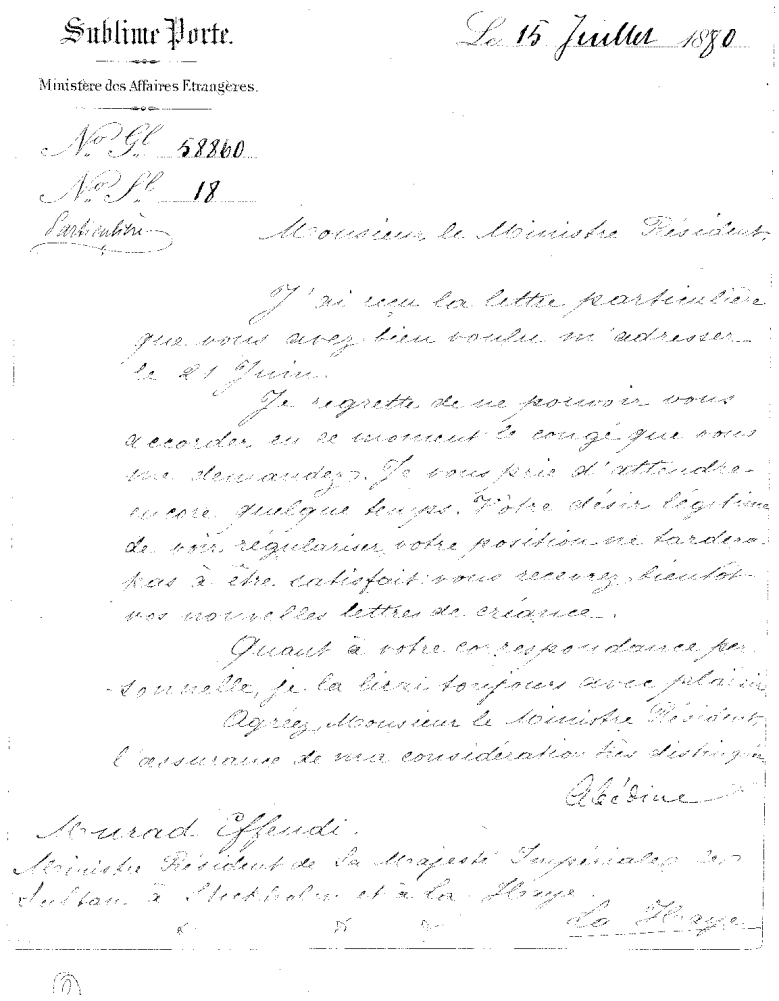

Texte 3 : Télégramme annonçant la déchéance du Sultan Abdülhamit et son remplacement par Mohammed V

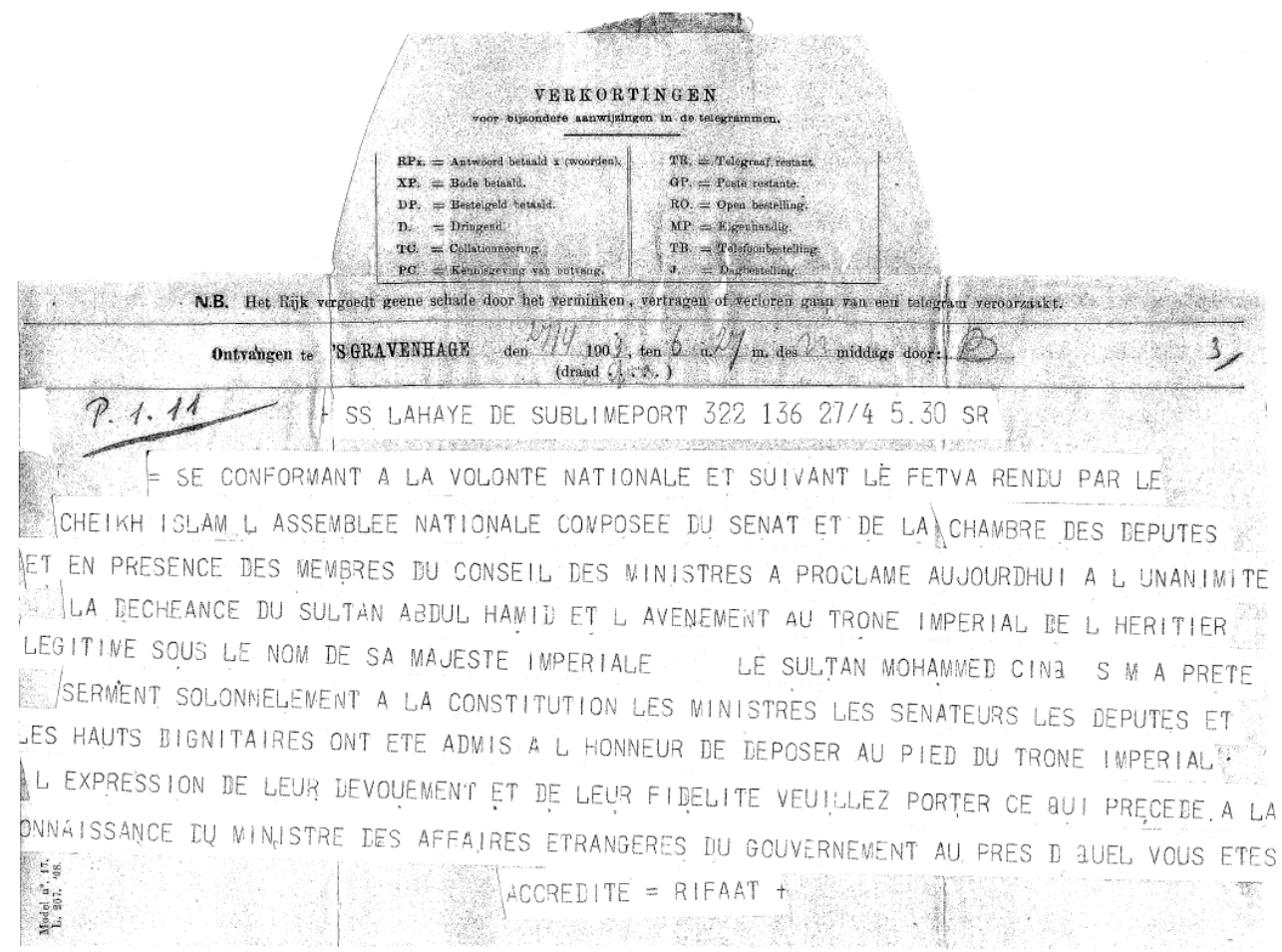


Texte 4 : Lettre envoyée au ministre plénipotentiaire de La Haye (1921)

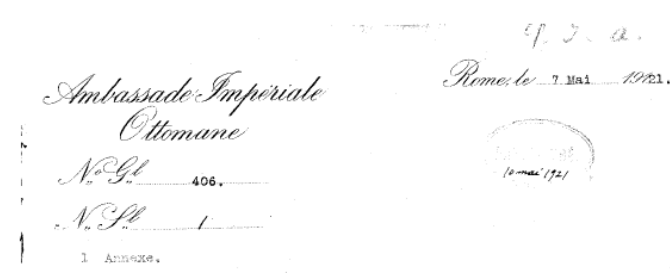

J'a1 1'honneur de transmattre ci-gròs à Votz Excollenoe la coupuro d'uns lettrê adreaséé de Plréa par une personne de confianoe à $\mathrm{S}$ sll. Calib Kémaly Bey, at d'où il reg-

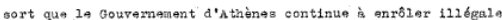
ment 198 graos originaires des régions ocoupées actuallement

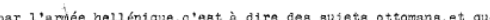

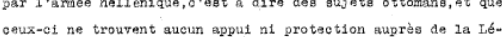
gation Royale de Hollande qui eat 'ohargée de la ssuvegarde des intérâta ottomang an Grò̀ce.

Du reste oes derniars jours plusieurs greos-

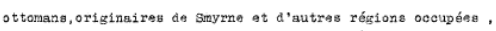
Be sont acressés is 1 'Ambagsade Impériale pour avoir des passeports at ont déolaré que, ne rouzant point servir dens I'armée

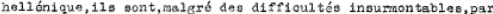
7anus à s'enfuir et à aq réfigior an Italia.

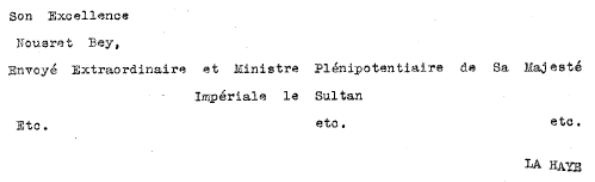

Texte 5 : Erched Bey, consul général ottoman à Batavia à Aristarchi Bey, légat ottoman à La Haye

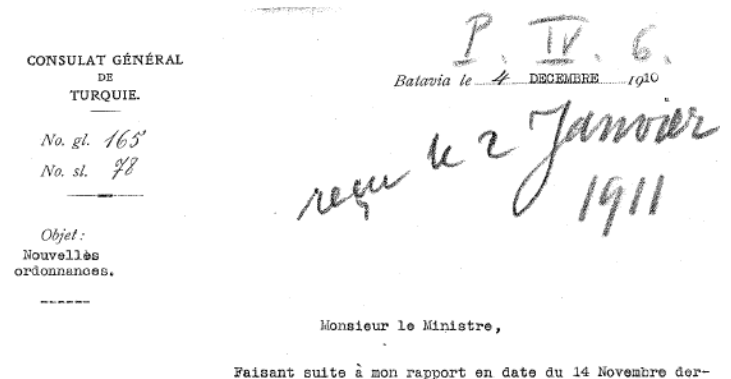
nitor a d'après un avis publí hier soir dans le presse, Honsiour lo Gouver neur Général a déja transmis. les ordres nécessaires aux autoríté̉ provinoiales pour commencer à mettro en pratique, à partir du premi. Janvier prochain, les nouvelles oráonnances sur le sajour et voyage des ótrangers orientaux dane 1 en 1198 de Java ot do lacaura. Vouillez agréer, Nonsiour le Hinistre, 1'assuranoe de la haute oonsidóration aveo laquelle J'al 1 'honneur d'ŝtro, de Votre Exoellenoe, le très humble et très obáïssant servitour.

$$
\text { Sucked. }
$$

Son Exoellenoe,

ARISTARCHI BEY,

$$
\begin{array}{cccc}
\text { II I N I S TRE } & \text { DI T URQUI } \\
\text { eto } & \text { oto } & \text { etc. }
\end{array}
$$


Texte 6 : Assim Bey, ministre ottoman des Affaires étrangères, au chargé des affaires au sujet de Madame Mourad Effendi

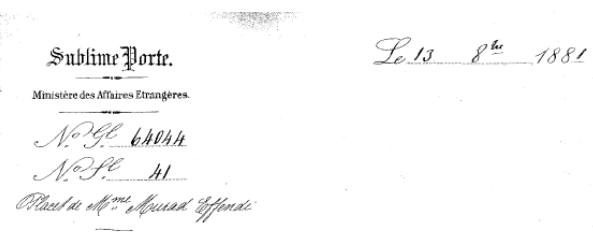

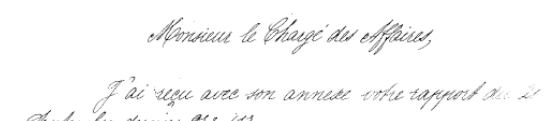

$$
\begin{aligned}
& \text { detembre demier, \%5: bt: } \\
& \text { Le rlacet de chloadanne ifferuad Ifferraci a ife } \\
& \text { communiqui au Grand effarichal du pratuis poru ithe }
\end{aligned}
$$

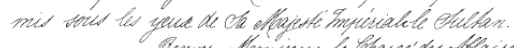

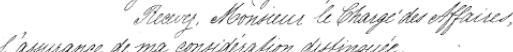

$$
\begin{aligned}
& \text { M. } \sin
\end{aligned}
$$

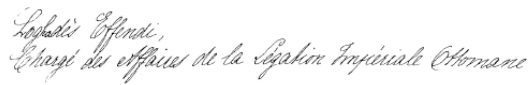

$$
\begin{aligned}
& \text { la Heage. }
\end{aligned}
$$

Texte 7 : Talaat Bey, chargé d'affaires, à Said Halim Pacha, ministre des Affaires étrangères

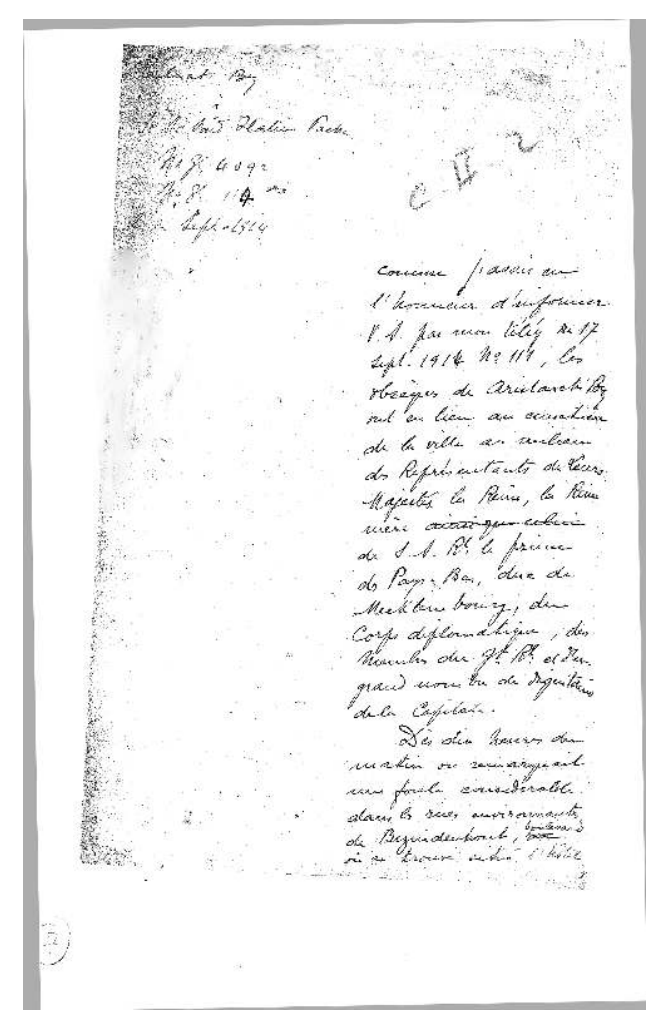




\section{Texte 8 : Note verbale destinée à la légation ottomane}

CABINET

DU MINISTRE.

No. 1561.

Le Ministère des Affaires itrangères a l'honneur

a'accuser réception d la Légation ottonane de la note du 14 de ce mois, No. 7247 , transmettant au Ministère cojie d'une note que le Gouvernement Irapérial vient d'adresser aux Hauts-Comissariats de Yrance, de Gande-Bretagne et a'Italie a constantinople au sujet du traltement des musulmans en Thrace orlentale.

La Haye, le 22 mars, 1921.

Texte 9 : Le ministre néerlandais des Affaires étrangères à Aristarchi Bey

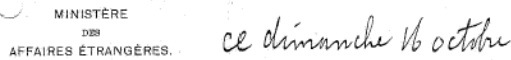

Mncher Ministre,

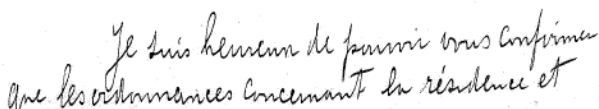

In lhe cereulition des ovientance" duns noo

alonies ant ide promulynés et seront paitheies

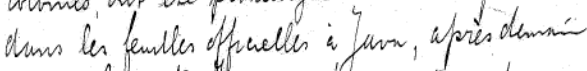

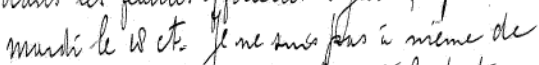

arso a procuner dis mantenant le lente enacti

Anons fint le tempos de le fair verin de

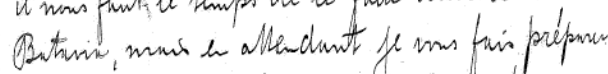

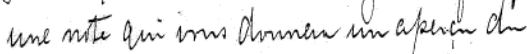

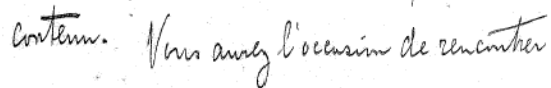


Jendition chy moi an doner diaher pom

Dir Gerge Danchume el anquel ansinary

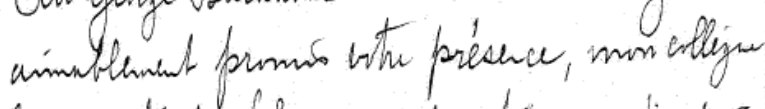

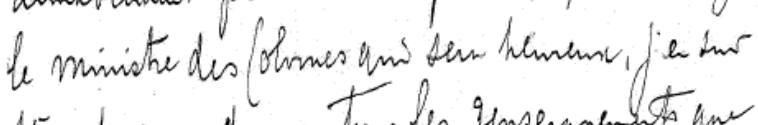

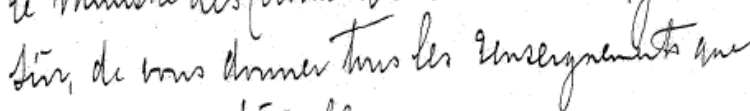

tons Juging désinbles

Bemile apeew, mancher thisite, en

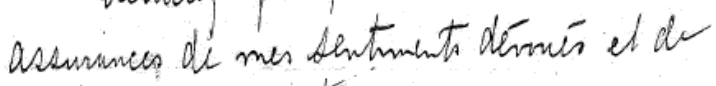

ma vioule considérition.

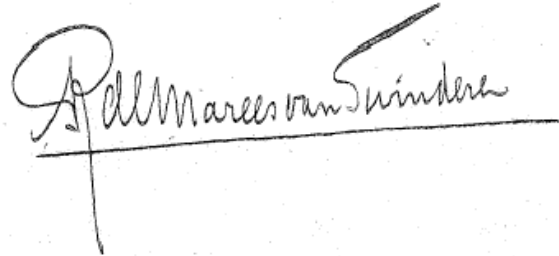

Texte 10 : Contrat de location entre le personnel de la légation et les propriétaires néerlandais

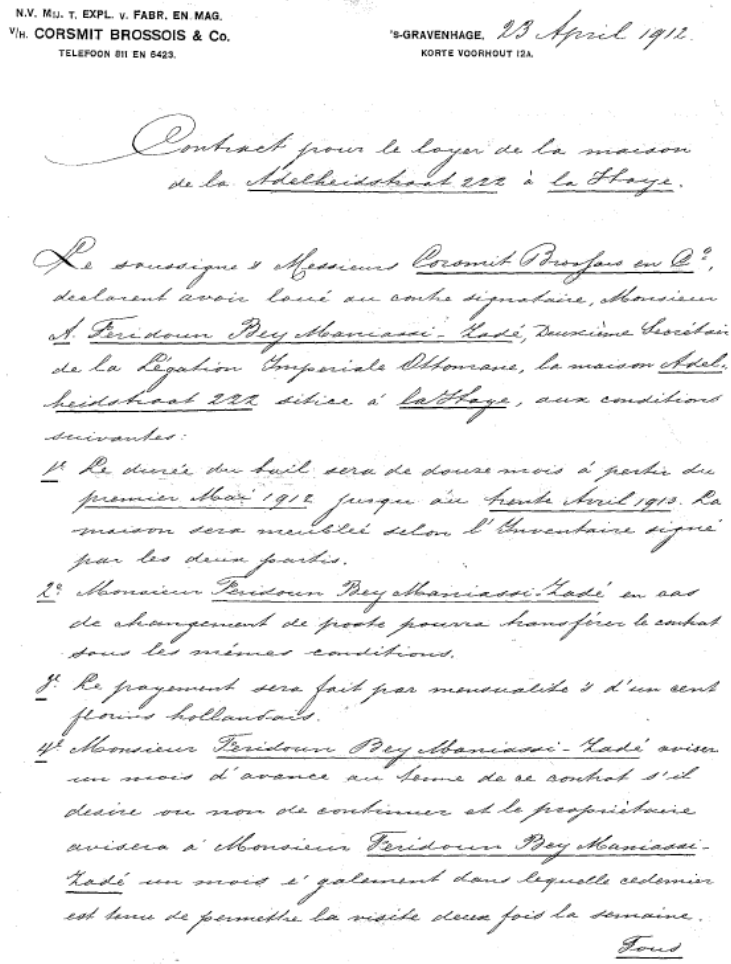


Texte 11 : Avis de recrutement aux sujets turcs

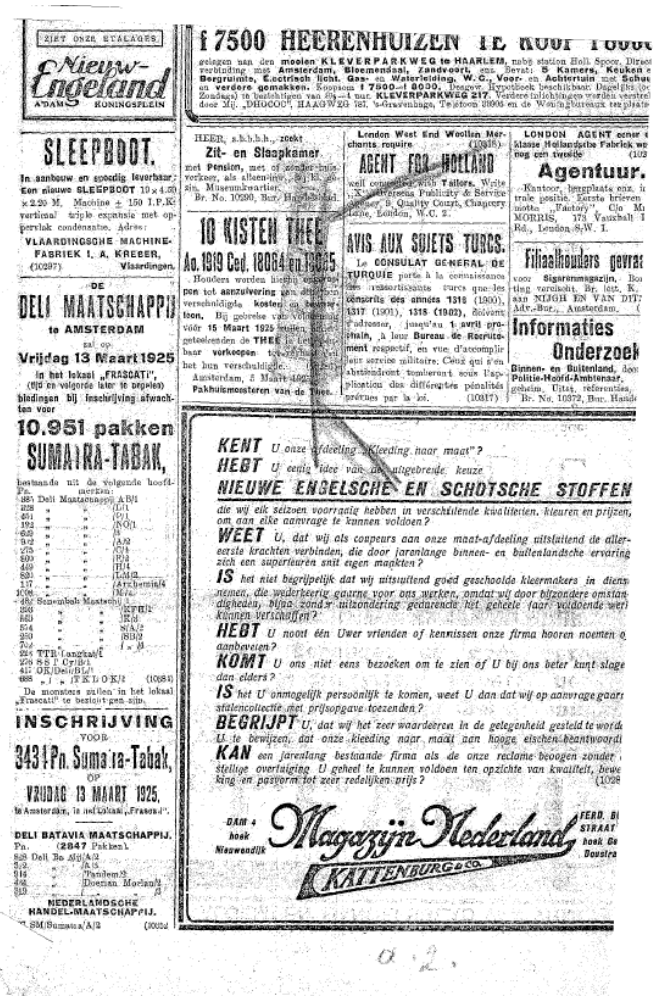

Texte 12: Annonce d'amnistie accordée aux déserteurs de la Grande Guerre

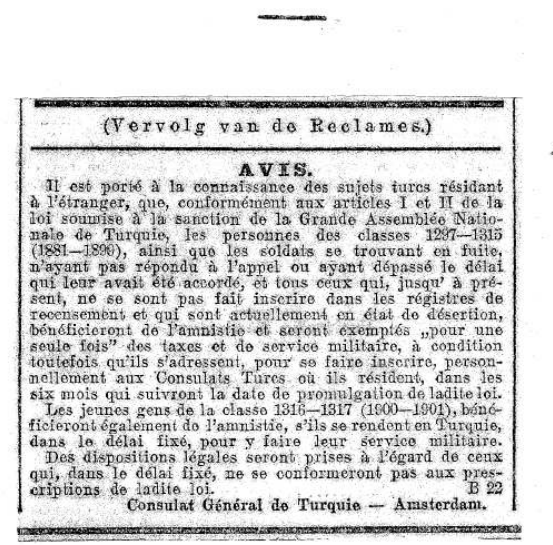


Texte 13 : Règlement bilingue des passeports des ressortissants ottomans

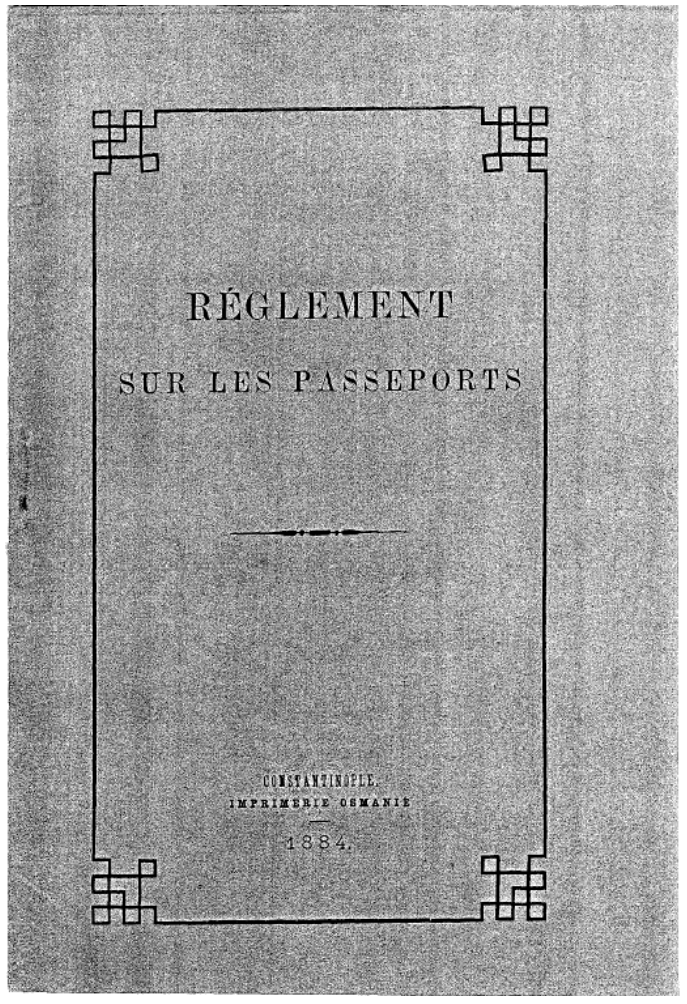

Texte 14 : Certificat de nationalité bilingue (19

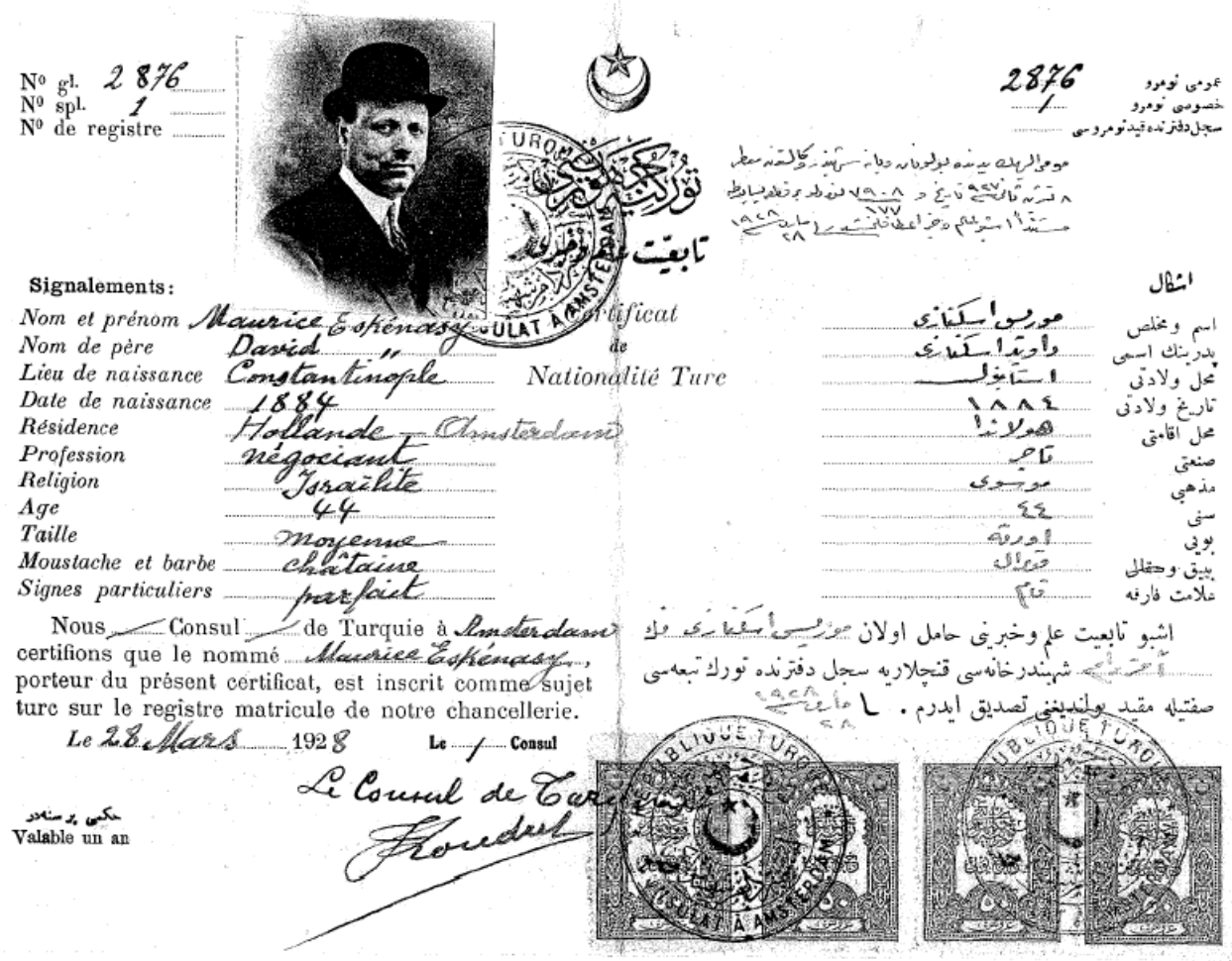




\section{BIBLIOGRAPHIE}

ARISTARCHI BEY (1873-1878). Législation ottomane. Istanbul : Imprimerie Frères Nicolaïdes.

ETENSEL ILDEM, A. (1997). « Les traductions littéraires du français en turc : aperçu historique et tendances actuelles ", in La Traduction carrefour des cultures et des temps. Istanbul : université Technique de Yildiz.

MEYDAN, S. (2006). Son Truvalılar. Istanbul : Truva Yayınları.

\section{NOTES}

1. Voir un excellent livre sur les relations diplomatiques entre l'Empire ottoman et les Pays-Bas en éditionbilingue : Çelikkol, Z. \& De Grott, A. (2000) ... Lâle ile Başladı/... It Bigan with the Tulip. Ankara : Türk Tarih Kurumu.

\section{RÉSUMÉS}

La correspondance diplomatique entre la Sublime Porte et la légation ottomane de La Haye qui a été établie dans les années 1860 se faisait en partie en français tandis que les échanges entre les autorités néerlandaises et la légation ottomane se réalisaient presque entièrement en français. Dans les années qui suivent la Première Guerre mondiale, la légation ottomane de La Haye publie en français dans les journaux néerlandais des annonces pour les sujets ottomans. Les contrats des secrétaires néerlandais de la légation sont rédigés en français. Nous pouvons multiplier les exemples. Le but de ce travail est d'étudier dans une perspective diachronique, le rôle que la langue française a joué dans la diplomatie ottomane. Cette étude se base, entre autres, sur les archives de l'Ambassade de Turquie à La Haye.

The diplomatic correspondence between the Sublime Porte and the Ottoman Legation of The Hague, established in 1860, was held partly in French where as the exchanges between the Dutch authorities and the Ottoman Legation was done almost totally in French. During the years following the First World War the Ottoman Legation of The Hague published in the Dutch newspapers announcements in French for the Ottoman citizens. The contracts of the Dutch secretaries were written in French. We can multiply the examples. The aim of this article is to study in a diachronic perspective the role played by French language in the Ottoman diplomacy. This study is based on the archives of the Turkish Embassy in The Hague. 
INDEX

Keywords : diplomatic correspondence, Dutch newspapers, French language, history, Ottoman diplomacy

Mots-clés : correspondance diplomatique, diplomatie ottomane, histoire, journaux néerlandais, langue française

\section{AUTEUR}

\section{ARZU ETENSEL ILDEM}

Université d'Ankara, Turquie 